\title{
IL-33 enhances glioma cell migration and invasion by upregulation of MMP2 and MMP9 via the ST2-NF-кB pathway
}

\author{
JIAN-FEI ZHANG ${ }^{1 *}$, PENG WANG $^{2 *}$, YU-JIN YAN ${ }^{1}$, YONG LI $^{1}$, \\ MIN-WU GUAN $^{1}$, JIN-JUN YU ${ }^{1}$ and XIN-DONG WANG ${ }^{1}$ \\ ${ }^{1}$ Department of Neurosurgery, Affiliated Hospital of School of Medicine, Ningbo University, \\ Ningbo, Zhejiang 315211; ${ }^{2}$ Department of Neurosurgery, Nanjing Medical University \\ Affiliated Wuxi Second Hospital, Wuxi, Jiangsu 214002, P.R. China
}

Received March 23, 2017; Accepted August 14, 2017

DOI: $10.3892 /$ or.2017.5926

\begin{abstract}
As an important member of the interleukin (IL)-1 family, IL-33 plays a significant role in tumor progression. To explore this, we previously analyzed the association between IL-33 expression and the prognosis of patients with glioma. However, the function of the IL-33/ST2 axis in glioma remained unclear. In the present study, immunofluorescent staining results revealed that the expression levels of IL-33 and ST2 receptor in glioma tissues were higher than those in normal brain tissues. Invasion and migration assays demonstrated that IL-33 significantly increased glioma cell invasion and migration in vitro. Furthermore, knockdown of ST2 by siRNA attenuated the IL-33-induced increase in invasion and migration. In addition, ELISA results revealed that IL-33 upregulated the expression of matrix metalloproteinase (MMP)2 and MMP9. Western blot analysis results indicated that IL-33 stimulation increased the phosphorylation of nuclear factor $-\kappa \mathrm{B}(\mathrm{NF}-\kappa \mathrm{B})$ in a time- and dose-dependent manner. Moreover, silencing of the NF- $\kappa \mathrm{B}$ pathway by BAY 11-7082 resulted in the inhibition of IL-33induced invasion and migration, as well as the downregulation of MMP2 and MMP9 production. These findings indicate that IL-33 may be involved in the process of glioma cell invasion and migration by upregulating MMP2 and MMP9 via the ST2-NF- $\kappa$ B signaling pathway. Thus, IL-33 may be a novel therapeutic target for glioma.
\end{abstract}

\section{Introduction}

Glioma is the most frequent intracranial tumor in adult humans (1). Despite numerous clinical advances, the median

Correspondence to: Dr Xin-Dong Wang, Department of Neurosurgery, Affiliated Hospital of School of Medicine, Ningbo University, 247 Renmin Road, Ningbo, Zhejiang 315211, P.R. China

E-mail: 15195962353@163.com

"Contributed equally

Key words: IL-33, NF-кB, glioma, invasion, migration survival time for high-grade glioma remains poor (2), primarily due to the invasive nature of glioma within the central nervous system (CNS). Therefore, the mechanisms regulating the aggressive characteristics of glioma are logical therapeutic targets.

The invasiveness of glioma cells is partly regulated by the interaction of cells with the extracellular matrix (ECM), followed by the degradation of the ECM by tumor cellderived proteases, particularly matrix metalloproteinases (MMPs) (3-6). MMPs are the principal secreted proteinases required for ECM degradation during tumor invasion and metastasis $(7,8)$. The overexpression of MMPs is well documented in malignant gliomas. Among the MMPs, the present study focused on MMP2 and MMP9, which are considered to enhance the invasion of glioma (9-11). However, the molecular regulation of MMP2 and MMP9 in glioblastoma has not been fully clarified.

The glioma microenvironment, including glial cells and their inflammatory products, regulates tumor development and progression (12). As a member of the interleukin (IL)-1 superfamily of cytokines, IL-33 is a multi-functional proinflammatory cytokine that is released upon cell damage and serves as an 'alarm' (13). Live cells can also produce IL-33 under biomechanical stress conditions (14). IL-33 is initially generated as a precursor protein that can be digested into a secreted and mature form by caspase-1 (15). IL-33 can bind to the receptor ST2 and induce the activation of various signaling proteins, including $\mathrm{p} 38$, nuclear factor- $\kappa \mathrm{B}(\mathrm{NF}-\kappa \mathrm{B})$, c-Jun N-terminal kinase (JNK) and extracellular signalregulated kinase $1 / 2(E R K 1 / 2)$, which promote the production of pro-inflammatory cytokines, such as IL-1, IL-6, tumor necrosis factor (TNF) and chemokine (C-C motif) ligand 2 (CCL2) (16-18). In addition to the pro-inflammatory cytokines, the IL-33/ST2 axis can also increase the expression of MMP2, MMP3 and MMP9 (19,20). Recent studies have reported that a high level of IL-33 is a diagnostic and prognostic marker for hepatocellular carcinoma and non-small cell lung cancer $(21,22)$. The IL-33/ST2 axis can also facilitate gastric cancer migration and invasion by inducing the secretion of IL-6 and MMP3 (20). Accumulating data indicate the importance of the IL-33/ST2 axis in cancer growth. In the CNS, IL-33 was reported to be highly expressed in mature oligodendrocytes 
and gray matter astrocytes, and released at sites of injury to promote recovery (23). More recently, it was reported that IL-33 is highly expressed in glioma cells and facilitates cell proliferation and migration via the regulation of growth factor and chemokine expression (24). Previously, we detected the overexpression of IL-33 in glioma tissues compared with that in normal brain tissues, and identified IL-33 overexpression as an independent factor that predicted a poor prognosis in patients with glioma (25). However, the underlying molecular mechanisms of the IL-33/ST2 axis during glioma development remain to be elucidated.

In the present study, the effects of IL-33 on glioma cell invasion and migration were investigated, and the underlying mechanisms of its function were examined. The results revealed that IL-33 and ST2 expression levels were positively correlated with the tumor grade. In addition, IL-33 promoted glioma cell migration and invasion via ST2. Furthermore, we found that the IL-33/ST2 axis induced increases in MMP2 and MMP9 through activation of NF- $\mathrm{B}$ signaling. Collectively, these findings suggest that IL-33 increases glioma cell migration and invasion by stimulating the secretion of MMP2 and MMP9 via the ST2-NF- $\kappa$ B pathway. Therefore, IL-33 may be an effective therapeutic target for the treatment of glioma.

\section{Materials and methods}

Cell culture, reagents and tumor samples. U87 and U251 cell lines were cultivated in DMEM (Gibco, UK) supplemented with $10 \%$ fetal bovine serum (FBS; Hyclone, Logan, UT, USA) and $0.05 \%(\mathrm{v} / \mathrm{v})$ gentamycin (Gibco) in petri dishes at $37^{\circ} \mathrm{C}$ and $6 \% \mathrm{CO}_{2}$. Recombinant human IL-33 was purchased from R\&D Systems (Minneapolis, MN, USA). Anti-IL-33 antibody (\#ABF108) was purchased from Millipore (Billerica, MA, USA), and anti-ST2 antibody (\#ab25877) was purchased from Abcam (Cambridge, MA, USA). A specific antibody against phospho-NF-кB (\#3033) was obtained from Cell Signaling Technology, Inc. (Beverly, MA, USA). An inhibitor of the $\mathrm{NF}-\kappa \mathrm{B}$ signaling pathway (BAY 11-7082) was obtained from Abcam.

Glioma tissue sections were obtained from the Division of Neurosurgery, Wuxi Second People's Hospital Affiliated to Nanjing Medical University (Wuxi, Jiangsu, China). The glioma tissue collection was approved by the Institutional Review Board of Wuxi Second People's Hospital Affiliated to Nanjing Medical University. According to the WHO criteria, the pathological grades of the glioma specimens were grouped into the following two categories: low-grade (grades I-II; 18 cases); and high-grade (grades III-IV; 28 cases). In addition, 15 tumor-adjacent brain tissue samples and 16 normal brain tissue samples were included. This study was approved by the Ethics Review Committee of Wuxi Second People's Hospital Affiliated to Nanjing Medical University (permission no.: WXEY2013-0218).

Immunohistochemistry. According to the standard method, all tumor tissue sections were dewaxed. After preconditioning, slides were incubated with IL-33 and ST2 primary antibodies at $4^{\circ} \mathrm{C}$ overnight. The slides were subsequently incubated with secondary antibodies at $37^{\circ} \mathrm{C}$ for $30 \mathrm{~min}$. Distinct cytoplasmic staining of ST2 and IL-33 were considered to indicate positive immunoreactivity.

Immunofluorescent staining. The experimental procedure of immunofluorescent staining was performed as previously described (26). Glioma tissues were acquired following surgery and frozen immediately. The slices were incubated with the anti-IL-33 antibody at $4{ }^{\circ} \mathrm{C}$ overnight, followed by nuclear counter staining with DAPI (1:5,000, Sigma). Images were subsequently observed and photographed with a fluorescent microscope (Nikon, Tokyo, Japan).

Migration assay. Cell migration was determined using Transwell chambers (Corning Costar, San Diego, CA, USA). In brief, $200 \mu \mathrm{l}$ of cell suspension ( $1 \times 10^{5}$ cells) was added into the upper wells, and the lower wells were filled with DMEM as a chemoattractant. After incubation for $12 \mathrm{~h}$ at $37^{\circ} \mathrm{C}$, cells on the top surface were removed with a cotton swab, and cells on the lower surface were fixed in methanol, stained with crystal violet and counted under the microscope. Five predetermined fields were counted for each membrane, and the mean values from three independent experiments, performed in duplicate, were used. The data were presented as the mean \pm standard deviation.

Invasion assay. Cell invasion was also performed using Transwell chambers. Briefly, $1 \times 10^{5}$ cells in $0.2 \mathrm{ml}$ of serum-free media were seeded into the upper chambers of Matrigel-coated filters. The lower chambers were filled with $0.5 \mathrm{ml}$ of complete medium as a chemoattractant. After incubation at $37^{\circ} \mathrm{C}$ for $12 \mathrm{~h}$, cells in the upper chamber were removed with a cotton swab, and cells on the lower side of the chamber were fixed with methanol, stained with crystal violet, and counted under the microscope. The mean values from three independent experiments performed in duplicate were used.

Transfection of siRNA. Cells were transfected with siRNA using Lipofectamine 2000 (Invitrogen, Carlsbad, CA, USA). The ST2 siRNA was designed and obtained from Invitrogen with the following sequence: 5'-CCAGAAAGGCCUCUAGU UUUU-3'. For transfection with ST2 siRNA, cells were plated in 12-well plates. At $6 \mathrm{~h}$ after transfection, the medium was removed and replaced with fresh medium supplemented with $10 \%$ FBS. Cells were further incubated for $24 \mathrm{~h}$.

Western blot analyses. An equal amount of protein from each protein sample was loaded onto an SDS-PAGE gel, separated by electrophoresis, and transferred to a PVDF membrane (Bio-Rad, Hercules, CA, USA). The membranes were blocked for $1 \mathrm{~h}$ in $1 \%$ milk (Bio-Rad) in PBST $(0.1 \%$ Tween-20 in PBS), then incubated with the primary antibodies overnight at $4^{\circ} \mathrm{C}$. After three washes with PBST, the membranes were incubated with peroxidase-conjugated secondary $\operatorname{IgG}(\mathrm{H}+\mathrm{L})$ antibodies for $1 \mathrm{~h}$ at room temperature and washed three times with PBST. The bands were then detected using Supersignal West Pico chemiluminescent substrate (Thermo Scientific).

Enzyme-linked immunosorbent assay for the determination of MMP2 and MMP9. Cell supernatant was centrifuged at 
A IL-33
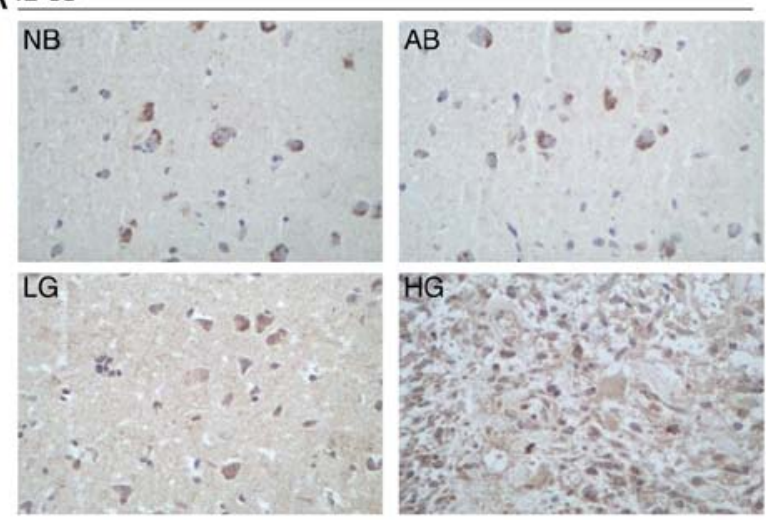

B ST2

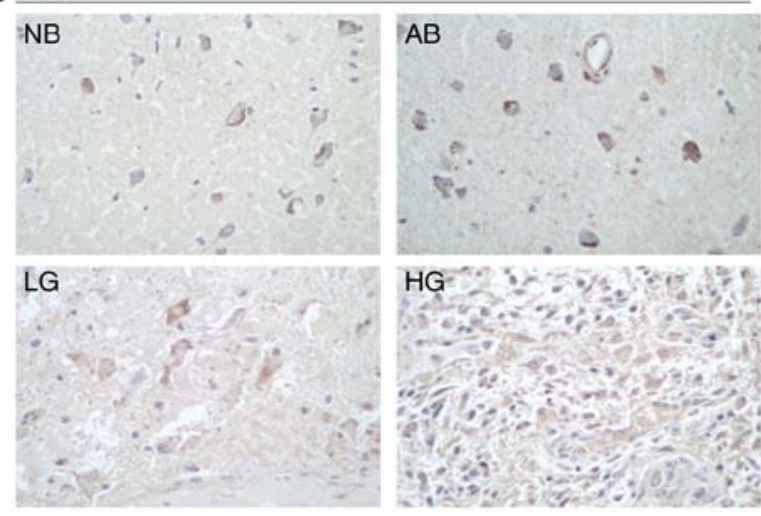

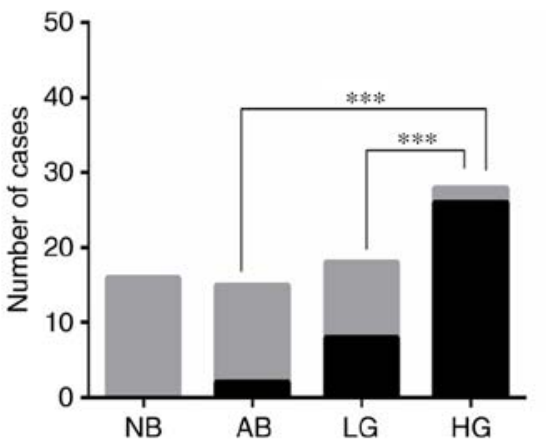

IL-33 low

IL-33 high

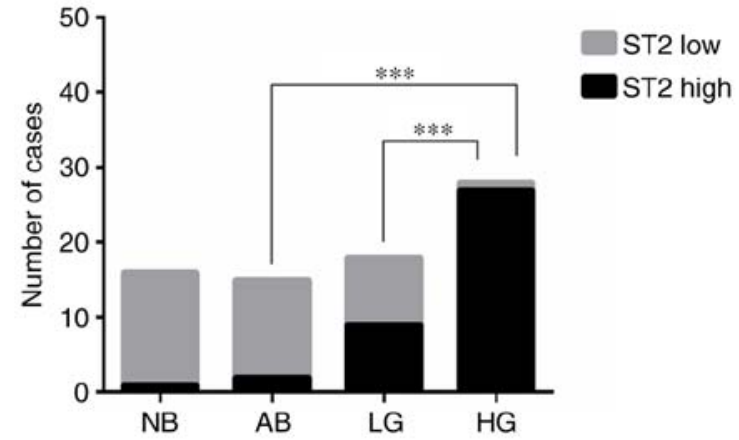

Figure 1. Immunohistochemical analysis of IL-33 and ST2 expression in normal brain tissue (NB), tumor-adjacent normal brain tissue (AB), low-grade glioma tissue (LG) and high-grade glioma tissue (HG). (A and B) Representative samples showing the results of immunohistochemical analysis in various tissues; IL-33 and ST2 expression were increased in glioma tissues compared with tumor-adjacent normal brain tissue (*** $\mathrm{p}<0.001$ for both). Magnification, $\mathrm{x} 400$.

$12,000 \mathrm{~g}$ for $15 \mathrm{~min}$ at $4^{\circ} \mathrm{C}$. MMP2 ELISA kits (RAB0365; Sigma, USA) and MMP9 ELISA kits (RAB0372; Sigma, Nanjing, China) were used to measure the levels of protein secretion of MMP2 and MMP9 by glioma cells, respectively, according to the manufacturer's instructions.

Statistical analysis. All data were presented as the mean \pm standard deviation. A Student's t-test was used to assess the statistical differences between two groups, and statistical differences among multiple groups were calculated by one-way analysis of variance. All data were analyzed using the statistical package GraphPad Prism version 5.0 (GraphPad Software Inc., La Jolla, CA, USA). p $<0.05, p<0.01$ and $p<0.001$ were considered statistically significant. All experiments were repeated at least three times.

\section{Results}

IL-33 and ST2 levels are elevated in glioma tissues and are associated with glioma grade. To determine the role of the IL-33/ST2 pathway in glioma tumorigenesis, we performed an immunohistochemical analysis of IL-33 and ST2 expression on samples of 15 tumor-adjacent normal brain tissues, 16 normal brain tissues, 18 low-grade glioma tissues and 28 high-grade glioma tissues. As shown in Fig. 1A, low IL-33 expression was observed in all 16 normal brain specimens and in 13 of the 15 tumor-adjacent normal brain specimens, whereas 8 of the
18 low-grade glioma specimens and 26 of the 28 high-grade glioma tissues expressed high levels of IL-33. ST2 expression was positively correlated with IL-33 expression (Fig. 1B). Moreover, IL-33 and ST2 proteins were detected in U251 and U87 cells by western blot analysis (Fig. 2A), and immunofluorescent staining revealed that IL-33 and ST2 were widely expressed in U87 glioma cells and glioma tissues (Fig. 2B and $\mathrm{C}$ ). Collectively, these results indicate that the expression levels of IL-33 and ST2 receptor in glioma tissues are higher than those in normal tissues, and that their expression levels are positively correlated with glioma grade.

IL-33 facilitates the invasion and migration of glioma cells in vitro. The association between IL-33/ST2 expression levels and glioma grade suggested that IL-33/ST2 may be important in migration and invasion. To examine the effects of IL-33 on cell invasion and migration, Transwell assays were performed. Glioma cells were treated with various concentrations of recombinant human IL-33 $(0,10,20$ and $50 \mathrm{ng} / \mathrm{ml})$, then subjected to invasion and migration assays. As shown in Fig. 3, IL-33 stimulation promoted the invasion and migration of U251 and U87 cells in a dose-dependent manner. These results illustrate that IL-33 may be involved in glioma cell invasion and migration. U251 cells were selected for subsequent study.

ST2 mediates the invasion and migration of glioma cells induced by $I L-33$. In order to investigate the relationship 

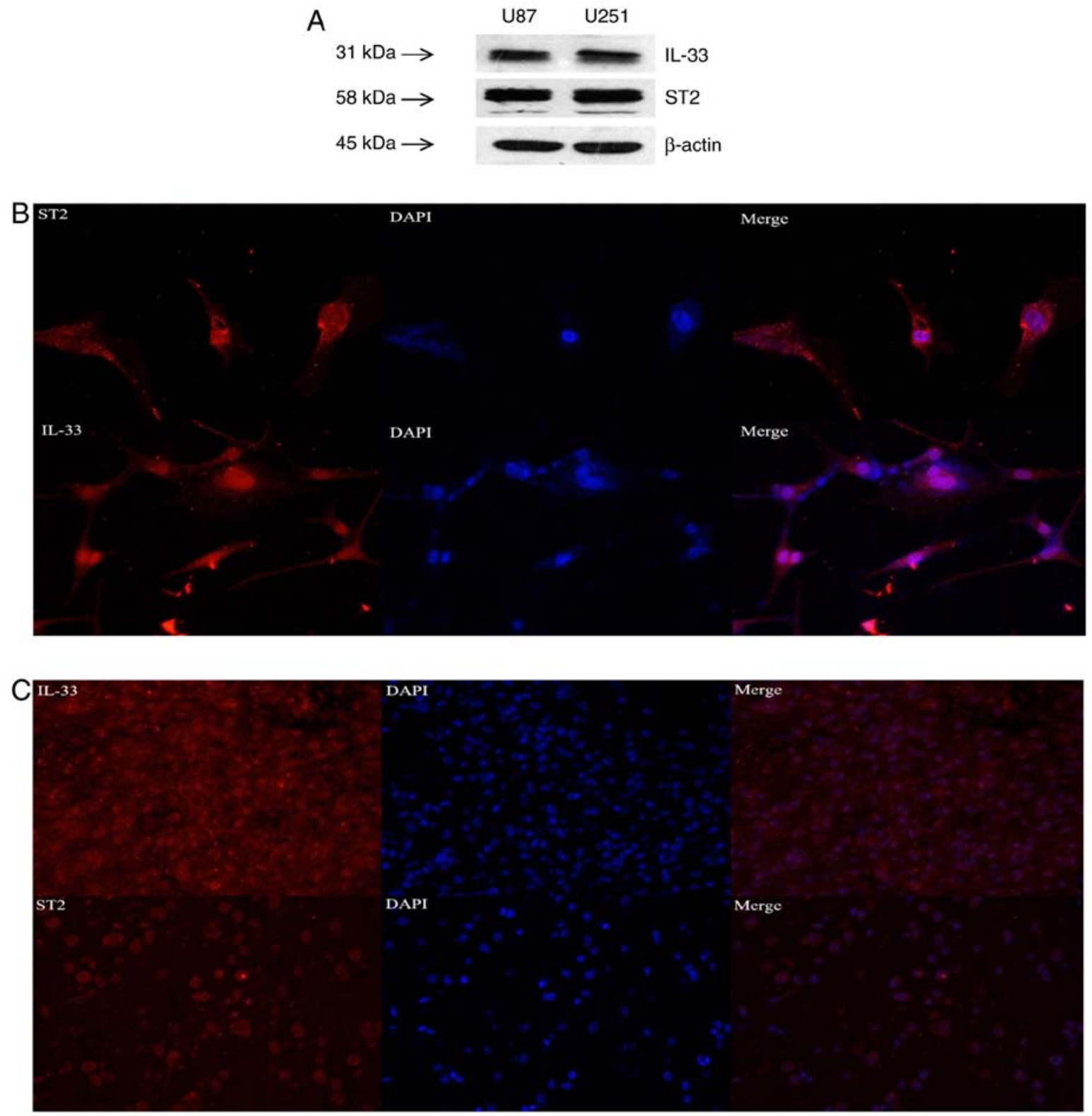

Figure 2. IL-33 and ST2 expression in glioma cells and tissues. (A) Western blot analysis of IL-33 and ST2 expression in U87 and U251 glioma cells. (B and C) Immunofluorescent staining for IL-33 and ST2 (red) and DAPI nuclear staining (blue) in U87 cells and glioma tissues.

between ST2 and IL-33-mediated invasion and migration in glioma cells, siRNA technology was used. U251 glioma cells were transiently transfected with ST2 siRNA or control siRNA (Con siRNA). Compared with Con siRNA-transfected cells, the expression of ST2 was dramatically inhibited in ST2 siRNA-transfected cells (Fig. 4A). Furthermore, the cells transfected with ST2 siRNA exhibited a distinct reduction in IL-33-induced invasion and migration compared with Con siRNA cells (Fig. 4B). These data suggest that ST2 plays an important role in IL-33-induced cell invasion and migration.

IL-33 upregulates the expression of MMP2 and MMP9 in glioma cells via the IL-33/ST2 pathway. IL-33 stimulation can induce the expression of MMPs $(19,20)$. In the present study, the expression levels of MMP2 and MMP9 were detected in glioma cells by ELISA following IL-33 treatment. The data indicated that IL-33 treatment led to significant increases in
MMP2 and MMP9 levels in the culture supernatant of U251 cells (Fig. 5A). Furthermore, to determine the involvement of ST2 in IL-33-stimulated secretion of MMP2 and MMP9, the protein levels of MMP2 and MMP9 in ST2-knockdown cells were examined after IL-33 stimulation for $20 \mathrm{~h}$, which illustrated that downregulation of ST2 decreased the expression of MMP2 and MMP9 compared with IL-33 stimulation alone (Fig. 5B). These results suggested that IL-33-mediated expression of MMP2 and MMP9 may depend on the IL-33/ST2 pathway.

IL-33 induces activation of the $N F-\kappa B$ pathway via the ST2 receptor. IL-33 can activate various signaling pathways, including NF- $\mathrm{B}, \mathrm{PI} 3 \mathrm{~K} / \mathrm{AKT}$ and MAPK/ERK, of which $\mathrm{NF}-\kappa \mathrm{B}$ was of particular interest in the present study. Recent studies have demonstrated that IL-33 can activate an NF- $\kappa \mathrm{B}$ kinase, leading to $N F-\kappa B$ phosphorylation, and drive the 

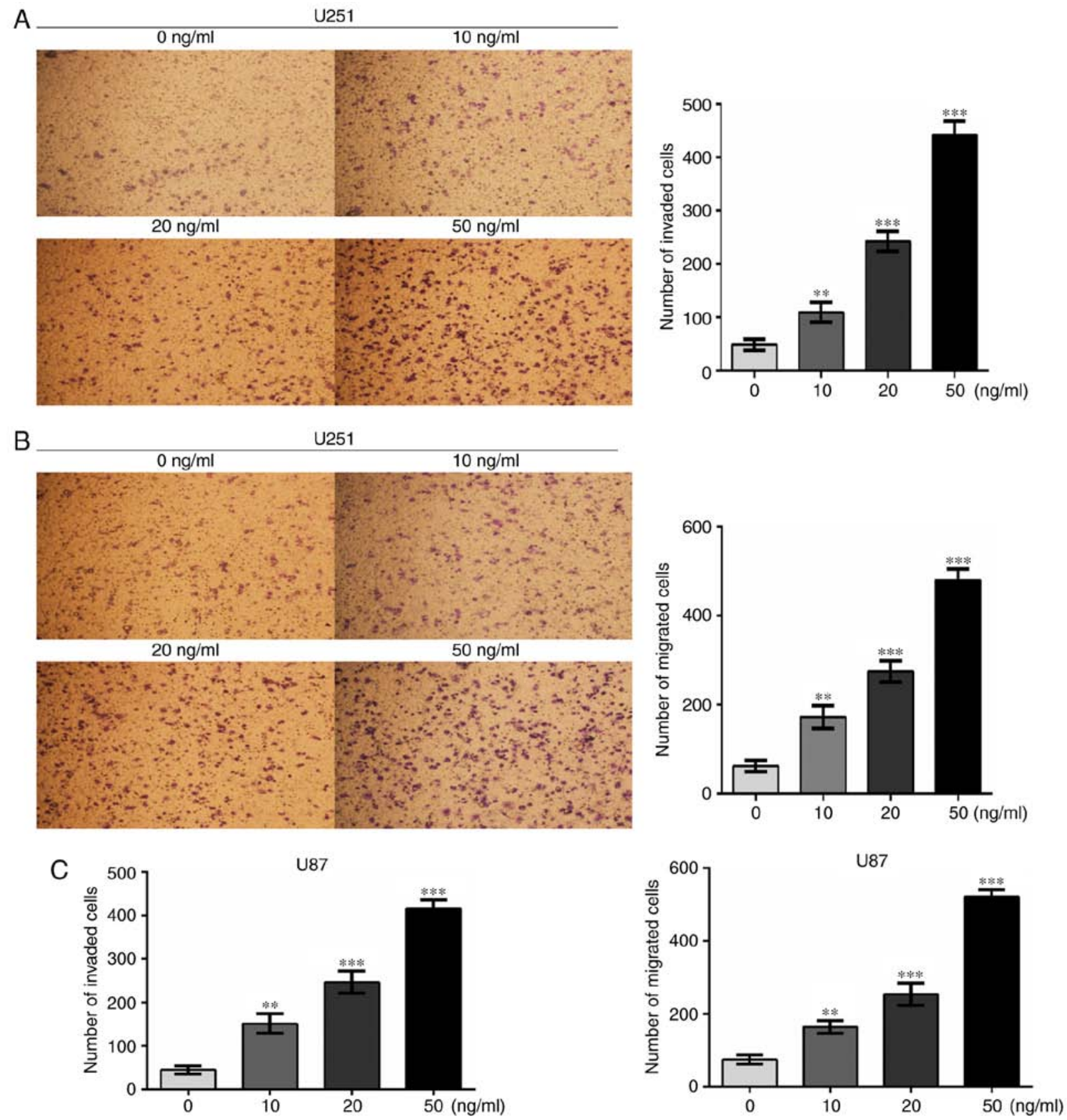

Figure 3. Effect of IL-33 on the invasion and migration of glioma cells. (A and B) Effects of IL-33 on the invasion and migration of U251 cells were evaluated by Transwell invasion and migration assays, respectively; magnification, x200. (C) Effects of IL-33 on the invasion and migration of U87 cells.** $\mathrm{p}<0.01$, **** $\mathrm{p}<0.001$.

production of Th2 cytokines via the ST2 receptor $(15,27)$. To investigate whether IL-33 may induce NF- $\mathrm{BB}$ activation in glioma cells, U251 cells were treated with $20 \mathrm{ng} / \mathrm{ml} \mathrm{IL-33} \mathrm{for}$ various durations. Western blot analysis indicated that IL-33 stimulation time-dependently enhanced the phosphorylation of NF- $\kappa \mathrm{B}$, with peak phosphorylation occurring at $30 \mathrm{~min}$ and decreasing levels at $60 \mathrm{~min}$ (Fig. 6A). Moreover, IL-33induced NF- $\mathrm{KB}$ kinase activation was significantly repressed in ST2 siRNA-transfected cells (Fig. 6B). These results indicate that IL-33/ST2 markedly induces the activation of NF- $\kappa B$ in glioma cells.

Effects of $N F-\kappa B$ pathway on IL-33-mediated glioma cell invasion, migration and secretion of MMP2 and MMP9.
To further elucidate the involvement of NF- $\mathrm{kB}$ signaling in the stimulation of MMP2 and MMP9 expression in glioma cells, the effect of a specific signal transduction inhibitor on glioma cells was examined. U251 cells were pretreated with BAY 11-7082 (an NF- $\mathrm{kB}$-specific inhibitor) for $30 \mathrm{~min}$ prior to IL-33 stimulation. Invasion and migration assays revealed that BAY 11-7082 suppressed IL-33-induced increases in the invasion and migration of $\mathrm{U} 251$ cells, indicating that the NF- $\mathrm{KB}$ pathway is required for the induction of invasion and migration by IL-33 (Fig. 7A and B). Furthermore, ELISA revealed that BAY 11-7082 decreased IL-induced secretion of MMP2 and MMP9, suggesting an involvement of the NF- $\kappa B$ pathway in regulating IL-33-induced secretion of MMP2 and MMP9 (Fig. 7C and D). 


\section{A Con siRNA ST2 SiRNA

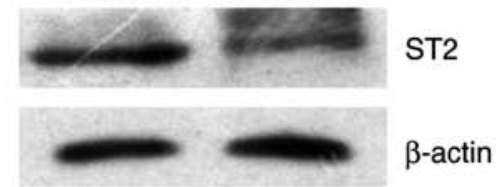

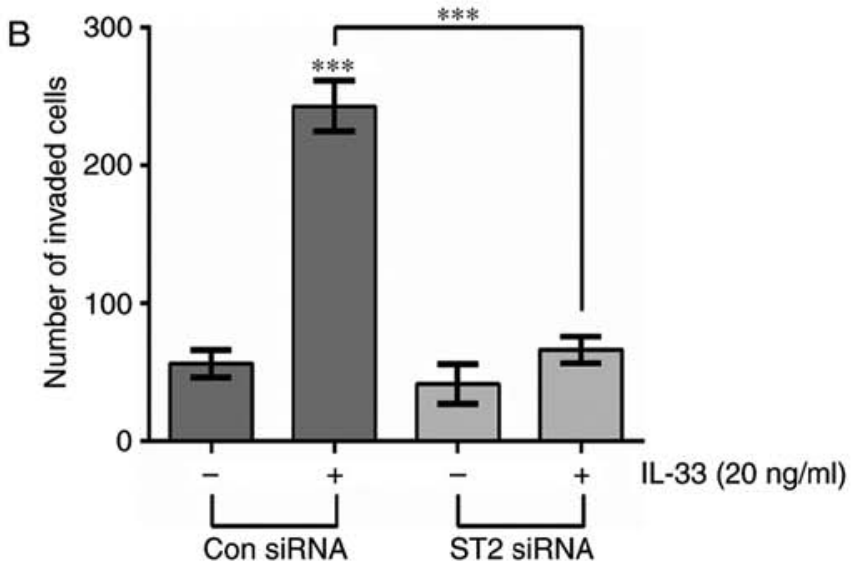
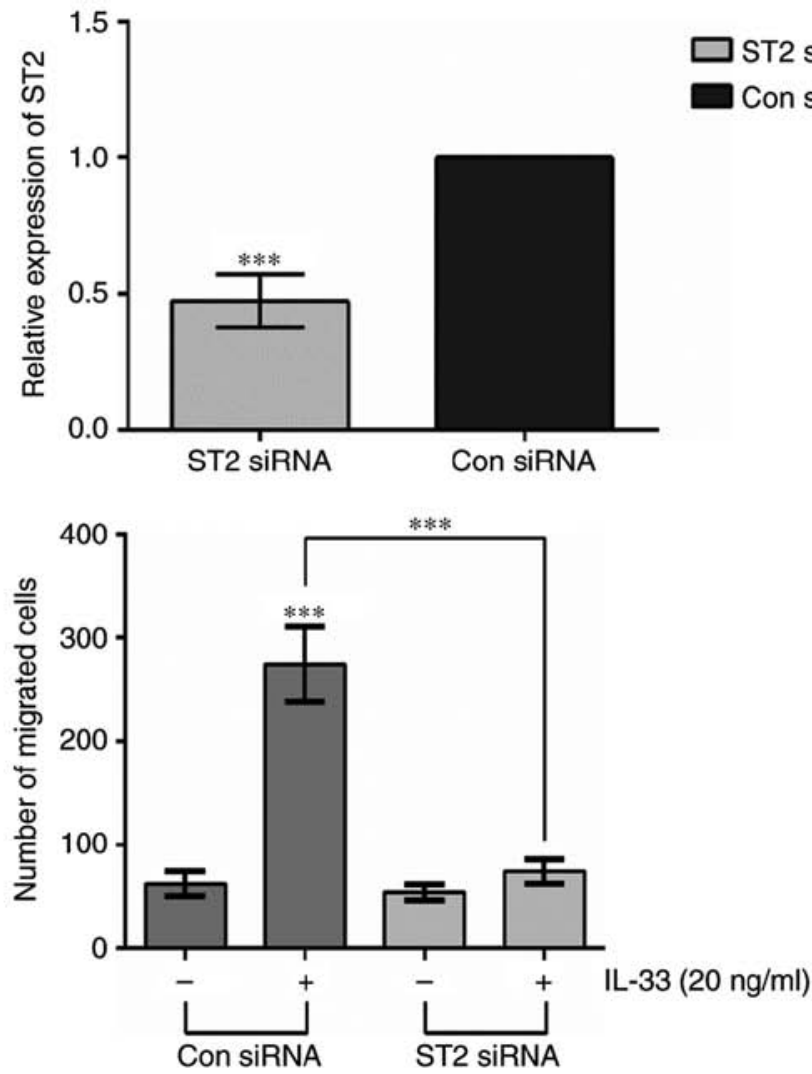

Figure 4. Effect of ST2 on IL-33-induced invasion and migration. (A) Western blot analysis indicated that knockdown of ST2 markedly inhibited the protein level of ST2. (B) Following the stimulation of cells with IL-33 (20 ng/ml), invasion and migration assays were performed. ${ }^{* * * *}$ p $<0.001$.

\section{A}

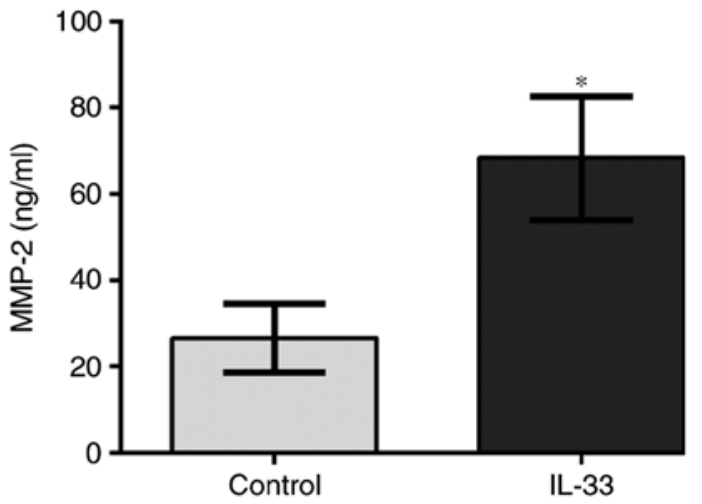

B

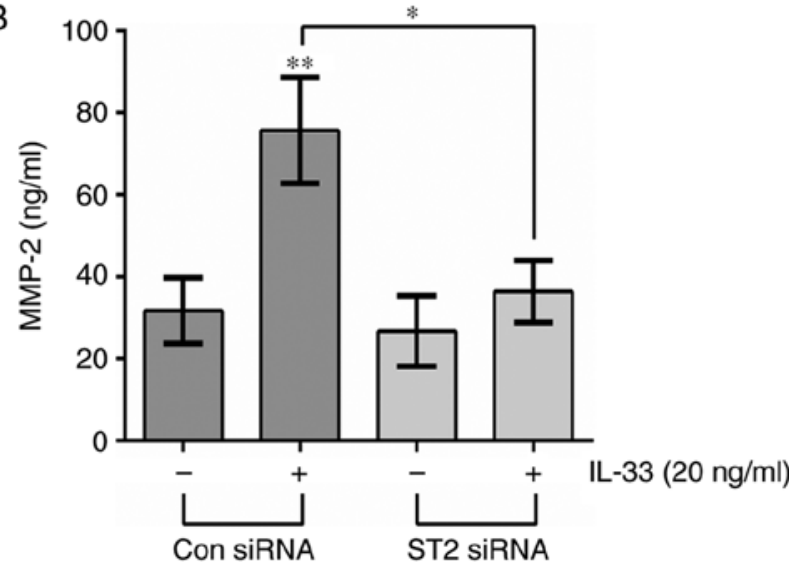

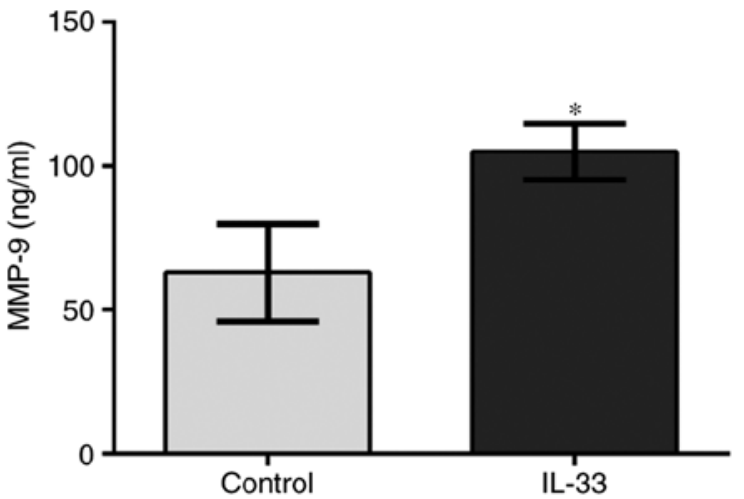

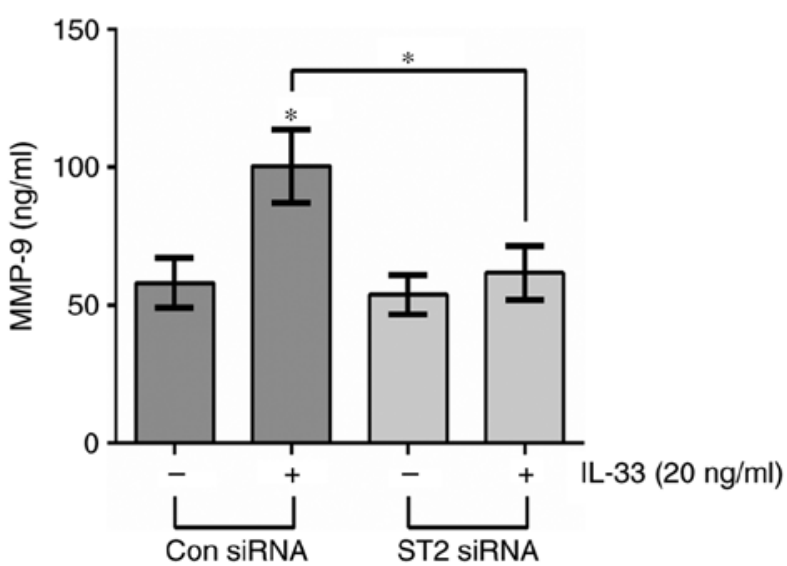

Figure 5. Effects of IL-33 and ST2 on the production of MMP2 and MMP9. (A) Following treatment with IL-33 (20 ng/ml) for 20 h, ELISA analysis was performed to determine the protein levels of MMP2 and MMP9 in the cell culture supernatant. (B) Following the knockdown of ST2 by a specific siRNA, cells were incubated with IL-33 and ELISA was performed to measure the protein levels of MMP2 and MMP9 in the cell culture supernatant. ${ }^{*} \mathrm{p}<0.05$, ${ }^{* *} \mathrm{p}<0.01$. 

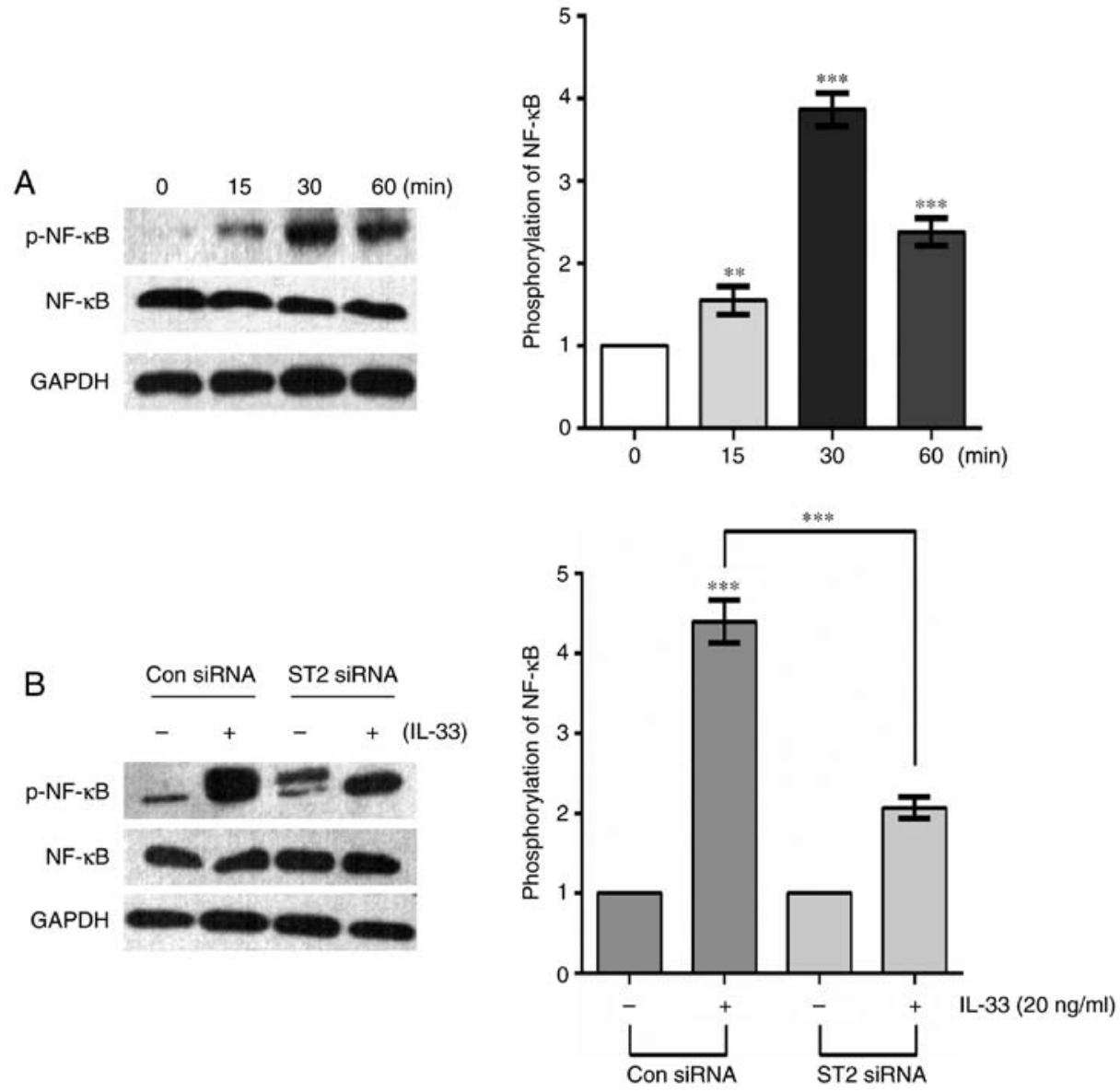

Figure 6. ST2 mediates IL-33-induced activation of NF-кB. (A) Western blot analysis was used to detect the phosphorylation of NF-кB in U251 cells. (B) Effect of ST2 knockdown on IL-33-induced NF-kB activation. ${ }^{* *} \mathrm{p}<0.01,{ }^{* * *} \mathrm{p}<0.001$.
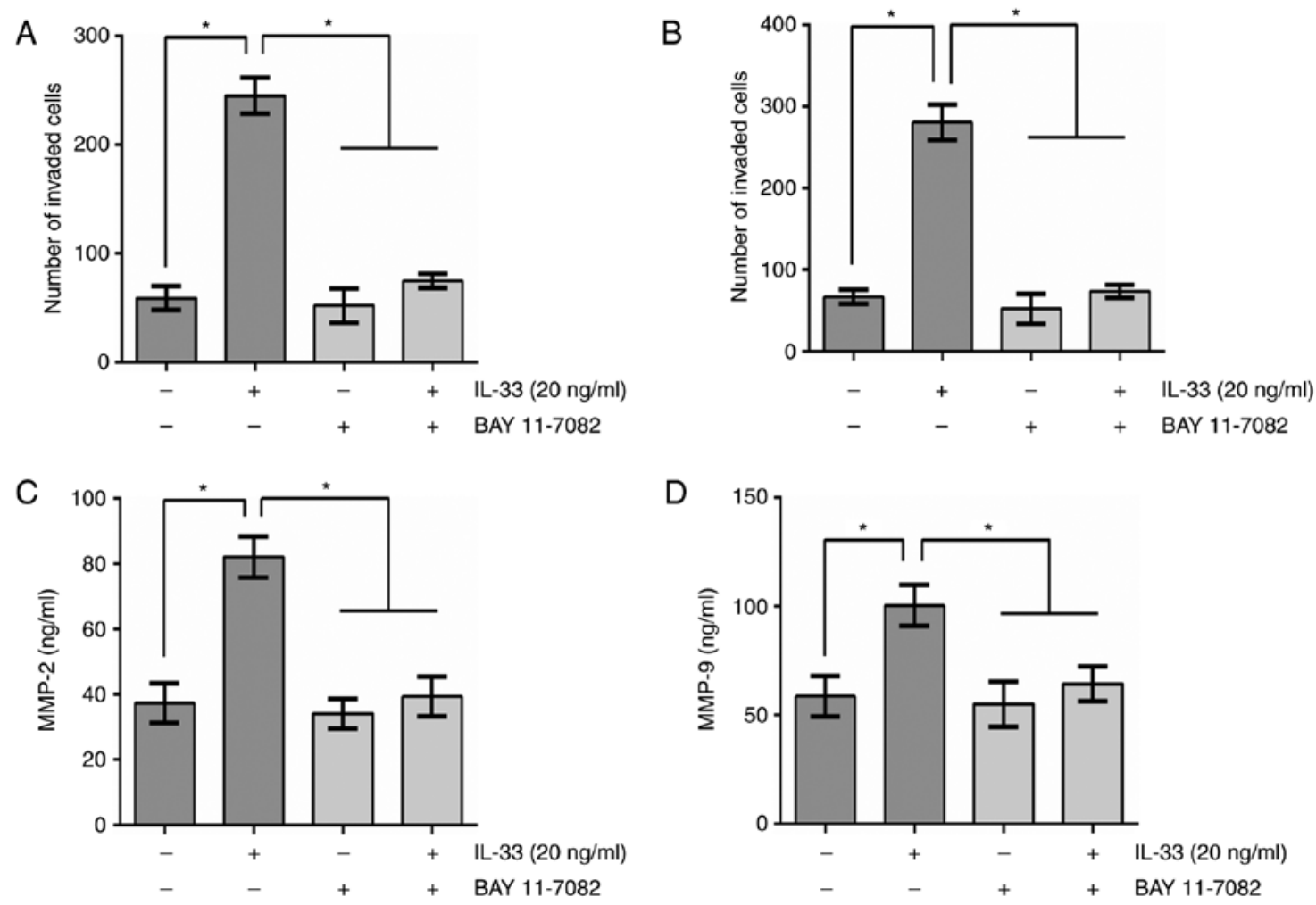

Figure 7. Effects of the NF-кB pathway on IL-33-mediated cell invasion, migration and secretion of MMP2 and MMP9. (A and B) Invasion and migration assays were performed on U251 cells that had been pretreated with BAY 11-7082 and stimulated with IL-33. (C and D) ELISA analysis revealed the protein levels of MMP2 and MMP9 in the cell culture supernatant. "p $<0.05$. 


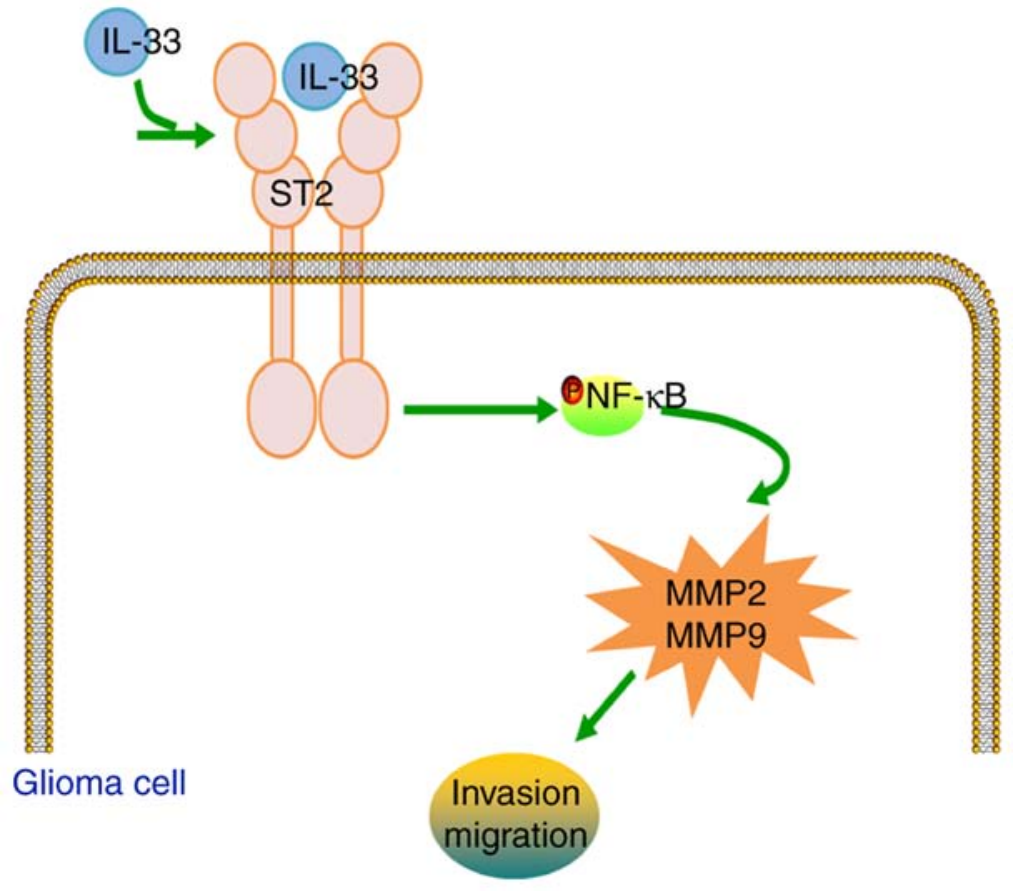

Figure 8. Schematic diagram of the role of IL-33 in the regulation of the biological behavior of glioma cells. IL-33 stimulates MMP2/MMP9 expression via the NF- $\mathrm{kB}$ signaling pathway, and enhances cell migration and invasion.

\section{Discussion}

The tumor microenvironment serves a crucial role in tumor development and progression (28). The tumor microenvironment consists of a variable combination of tumor cells, infiltrating leukocytes and endothelial cells, which produce cytokines and other substances that drive tumor development $(29,30)$. As a member of the IL-1 cytokine family, IL-33 has been shown to be involved in the progression and development of cancer (31). Elevated expression of IL-33 has been found in many types of tumors, including human colorectal, gastric and breast cancers $(19,20,32)$. Further studies have demonstrated that IL-33 increases the migration and invasion of gastric carcinoma (20). In the present study, the overexpression of IL-33 and ST2 was observed in glioma tissues. Importantly, we found that IL-33 can enhance the invasion and migration of glioma cell lines. These results suggest that the IL-33/ST2 signaling pathway may play an important role in glioma cells.

IL-33 acts via its receptor, ST2. Asa member of the IL-1 receptor family, ST2 has two types: a soluble secreted form (sST2) and a transmembrane, full-length form (ST2L) (33). Certain clinical studies have confirmed that serum sST2 is associated with the progression of hepatocellular carcinoma (21). Moreover, activation of ST2 by IL-33 enhances the metastasis of breast cancer (34). Recently, Fang et al (24) found that IL-33 was a crucial factor in tumorigenic glioma C6 cells, and inhibition of IL-33 gene expression suppressed the growth rate and colony formation of C6 cells. Furthermore, IL-33 was associated with C6 cell migration and the regulation of the expression of several chemokines and growth factors (24). In accordance with previous reports, the data from the present study demonstrated that IL-33 promoted the migration and invasion of glioma cells, while
ST2 knockdown attenuated this effect, further verifying the involvement of ST2 in cancer.

Aside from its roles in promoting cancer progression, IL-33 has been shown to be associated with antitumor immune responses during cancer development $(8,31,35,36)$. In animal models, IL-33 could activate $\mathrm{CD} 8^{+} \mathrm{T}$ and natural killer (NK) cells, and inhibit the progression and migration of tumors $(8,36)$. Conversely, other studies suggested that IL-33 could enhance type-2 immune responses, suppress NK cells and promote tumor development in tumor-bearing animals $(34,37-40)$. These data suggest that IL-33 may have both pro-tumor and antitumor effects during the development of different tumors.

As a multi-functional pro-inflammatory cytokine, IL-33 stimulation can induce the activation of various signaling pathways, including the PI3K/AKT, ERK1/2, and MAPK pathways, and this has been shown to be involved in the progression and development of cancer $(20,41)$. NF- $\kappa B$ is a key factor in inflammation and innate immunity. A recent study showed that IL-33 could induce the activation of the NF- $\mathrm{kB}$ pathway to enhance the invasiveness of decidual stromal cells via the upregulation of CCL2/CCR2 (17). Another study showed that NF- $\kappa B$ activation could mediate the effect of IL-33 on cytokine production in pancreatic carcinoma cells (42). Concordantly, the results from the present study demonstrated that IL-33 activates the ST2/NF- $\mathrm{B}$ pathway, which contributes to glioma cell motility and invasion.

Although growing evidence indicates a role for IL-33 in tumor progression, the potential underlying mechanism by which IL-33 facilitates cancer cell growth and invasion remains unclear. Glioma invasiveness is mediated in part by MMPs (43-45). Secreted proteases, such as MMP2, MMP7, MMP9, can digest the ECM, basal lamina, and cell adhesion proteins, thereby promoting cell invasion and 
tumor growth $(3,9)$. IL-33 stimulation can induce a train of pro-destructive molecules, including IL-6, CXCR4, MMP2, MMP3 and MMP9. In the present study, the results demonstrated that IL-33 induced the expression of MMP2 and MMP9 in glioma cells, whereas the silencing of ST2 or inhibition of the NF- $\kappa$ B pathway could strongly suppress the IL-33induced expression of MMP2 and MMP9, indicating that the ST2-NF- $\kappa$ B pathway is required for IL-33-mediated MMP2 and MMP9 production. As MMP2 and MMP9 are necessary for tumor invasion and migration, it is possible that IL-33 facilitates glioma cell invasion and migration via upregulating MMP2 and MMP9 production.

In conclusion, the present data demonstrate that IL-33 stimulation facilitates the invasion and migration of glioma cells through the ST2-NF- $\mathrm{B}$ pathway in vitro. Activation of the ST2-NF- $\kappa$ B pathway, and subsequently of MMP2 and MMP9 production, may potentiate the biological effects of IL-33 on glioma cells (Fig. 8). These results suggest that IL-33 plays a significant role in glioma cell invasion and migration, which could contribute to the elucidation of glioma pathogenesis and the development of novel therapeutics.

\section{References}

1. Robins HI, Peterson CG and Mehta MP: Combined modality treatment for central nervous system malignancies. Semin Oncol 30 (Suppl 9): 11-22, 2003.

2. Cloughesy TF, Cavenee WK and Mischel PS: Glioblastoma: From molecular pathology to targeted treatment. Annu Rev Pathol 9: 1-25, 2014.

3. Rao JS: Molecular mechanisms of glioma invasiveness: The role of proteases. Nat Rev Cancer 3: 489-501, 2003.

4. Van Meter TE, Rooprai HK, Kibble MM, Fillmore HL, Broaddus WC and Pilkington GJ: The role of matrix metalloproteinase genes in glioma invasion: Co-dependent and interactive proteolysis. J Neurooncol 53: 213-235, 2001.

5. Yong VW: Metalloproteinases: Mediators of pathology and regeneration in the CNS. Nat Rev Neurosci 6: 931-944, 2005.

6. Yong VW, Power C, Forsyth P and Edwards DR: Metalloproteinases in biology and pathology of the nervous system. Nat Rev Neurosci 2: 502-511, 2001.

7. Mignatti $P$ and Rifkin DB: Biology and biochemistry of proteinases in tumor invasion. Physiol Rev 73: 161-195, 1993.

8. Gao X, Wang X, Yang Q, Zhao X, Wen W, Li G, Lu J, Qin W, Qi Y, Xie F, et al: Tumoral expression of IL-33 inhibits tumor growth and modifies the tumor microenvironment through $\mathrm{CD}^{+}$ T and NK cells. J Immunol 194: 438-445, 2015.

9. Forsyth PA, Wong H, Laing TD, Rewcastle NB, Morris DG, Muzik H, Leco KJ, Johnston RN, Brasher PM, Sutherland G, et al: Gelatinase-A (MMP-2), gelatinase-B (MMP-9) and membrane type matrix metalloproteinase-1 (MT1-MMP) are involved in different aspects of the pathophysiology of malignant gliomas. Br J Cancer 79: 1828-1835, 1999.

10. Kargiotis O, Chetty C, Gondi CS, Tsung AJ, Dinh DH, Gujrati M, Lakka SS, Kyritsis AP and Rao JS: Adenovirus-mediated transfer of siRNA against MMP-2 mRNA results in impaired invasion and tumor-induced angiogenesis, induces apoptosis in vitro and inhibits tumor growth in vivo in glioblastoma. Oncogene 27 4830-4840, 2008

11. Shastry AH, Thota B, Arimappamagan A and Santosh V: P53 stratification reveals the prognostic utility of matrix metalloproteinase-9 protein expression in glioblastoma. Neurol India 63: 399-404, 2015

12. Heimberger $\mathrm{AB}$ and Sampson JH: Immunotherapy coming of age: What will it take to make it standard of care for glioblastoma? Neuro-oncol 13: 3-13, 2011.

13. Palmer G and Gabay C: Interleukin-33 biology with potential insights into human diseases. Nat Rev Rheumatol 7: 321-329, 2011.

14. Kakkar R, Hei H, Dobner S and Lee RT: Interleukin 33 as a mechanically responsive cytokine secreted by living cells. J Biol Chem 287: 6941-6948, 2012.
15. Schmitz J, Owyang A, Oldham E, Song Y, Murphy E, McClanahan TK, Zurawski G, Moshrefi M, Qin J, Li X, et al: IL-33, an interleukin-1-like cytokine that signals via the IL-1 receptor-related protein ST2 and induces T helper type 2-associated cytokines. Immunity 23: 479-490, 2005.

16. Ali S, Huber M, Kollewe C, Bischoff SC, Falk W and Martin MU: IL-1 receptor accessory protein is essential for IL-33-induced activation of T lymphocytes and mast cells. Proc Natl Acad Sci USA 104: 18660-18665, 2007.

17. Hu WT, Li MQ, Liu W, Jin LP, Li DJ and Zhu XY: IL-33 enhances proliferation and invasiveness of decidual stromal cells by up-regulation of CCL2/CCR 2 via NF- $\kappa \mathrm{B}$ and ERK1/2 signaling. Mol Hum Reprod 20: 358-372, 2014.

18. Moulin D, Donzé O, Talabot-Ayer D, Mézin F, Palmer G and Gabay C: Interleukin (IL)-33 induces the release of pro-inflammatory mediators by mast cells. Cytokine 40: 216-225, 2007.

19. Liu X, Zhu L, Lu X, Bian H, Wu X, Yang W and Qin Q: IL-33/ ST2 pathway contributes to metastasis of human colorectal cancer. Biochem Biophys Res Commun 453: 486-492, 2014.

20. Yu XX, Hu Z, Shen X, Dong LY, Zhou WZ and Hu WH: IL-33 Promotes gastric cancer cell invasion and migration via ST2-ERK1/2 pathway. Dig Dis Sci 60: 1265-1272, 2015.

21. Bergis D, Kassis V, Ranglack A, Koeberle V, Piiper A, Kronenberger B, Zeuzem S, Waidmann O and Radeke HH: High Serum levels of the interleukin-33 receptor soluble ST2 as a negative prognostic factor in hepatocellular carcinoma. Trans Oncol 6: 311-318, 2013.

22. Hu LA, Fu Y, Zhang DN and Zhang J: Serum IL-33 as a diagnostic and prognostic marker in non-small cell lung cancer. Asian Pac J Cancer Prev 14: 2563-2566, 2013.

23. Gadani SP, Walsh JT, Smirnov I, Zheng J and Kipnis J: The gliaderived alarmin IL-33 orchestrates the immune response and promotes recovery following CNS injury. Neuron 85: 703-709, 2015.

24. Fang KM, Yang CS, Lin TC, Chan TC and Tzeng SF: Induced interleukin-33 expression enhances the tumorigenic activity of rat glioma cells. Neuro-oncol 16: 552-566, 2014.

25. Zhang J, Wang P, Ji W, Ding Y and Lu X: Overexpression of interleukin-33 is associated with poor prognosis of patients with glioma. Int J Neurosci 127: 210-217, 2017.

26. Ii M, Nishimura H, Iwakura A, Wecker A, Eaton E, Asahara T and Losordo DW: Endothelial progenitor cells are rapidly recruited to myocardium and mediate protective effect of ischemic preconditioning via 'imported' nitric oxide synthase activity. Circulation 111: 1114-1120, 2005.

27. Funakoshi-Tago M, Tago K, Hayakawa M, Tominaga S, Ohshio T, Sonoda Y and Kasahara T: TRAF6 is a critical signal transducer in IL-33 signaling pathway. Cell Signal 20: 1679-1686, 2008.

28. Radisky DC and Bissell MJ: Cancer. Respect thy neighbor! Science 303: 775-777, 2004

29. Balkwill F, Charles KA and Mantovani A: Smoldering and polarized inflammation in the initiation and promotion of malignant disease. Cancer Cell 7: 211-217, 2005.

30. Coussens LM and Werb Z: Inflammation and cancer. Nature 420: 860-867, 2002.

31. Milovanovic M, Volarevic V, Radosavljevic G, Jovanovic I, Pejnovic N, Arsenijevic N and Lukic ML: IL-33/ST2 axis in inflammation and immunopathology. Immunol Res 52: 89-99, 2012.

32. Kim JY, Lim SC, Kim G, Yun HJ, Ahn SG and Choi HS: Interleukin-33/ST2 axis promotes epithelial cell transformation and breast tumorigenesis via upregulation of COT activity. Oncogene 34: 4928-4938, 2015.

33. Bergers G, Reikerstorfer A, Braselmann S, Graninger P and Busslinger M: Alternative promoter usage of the Fos-responsive gene Fit-1 generates mRNA isoforms coding for either secreted or membrane-bound proteins related to the IL-1 receptor. EMBO J 13: 1176-1188, 1994

34. Jovanovic IP, Pejnovic NN, Radosavljevic GD, Pantic JM, Milovanovic MZ, Arsenijevic NN and Lukic ML: Interleukin-33/ ST2 axis promotes breast cancer growth and metastases by facilitating intratumoral accumulation of immunosuppressive and innate lymphoid cells. Int J Cancer 134: 1669-1682, 2014

35. Jovanovic I, Radosavljevic G, Mitrovic M, Juranic VL, McKenzie ANJ, Arsenijevic N, Jonjic S and Lukic ML: ST2 deletion enhances innate and acquired immunity to murine mammary carcinoma. Eur J Immunol 41: 1902-1912, 2011.

36. Gao K, Li X, Zhang L, Bai L, Dong W, Gao K, Shi G, Xia X, Wu L and Zhang L: Transgenic expression of IL-33 activates CD8(+) T cells and NK cells and inhibits tumor growth and metastasis in mice. Cancer Lett 335: 463-471, 2013. 
37. Miller AM, Xu D, Asquith DL, Denby L, Li Y, Sattar N, Baker AH, McInnes IB and Liew FY: IL-33 reduces the development of atherosclerosis. J Exp Med 205: 339-346, 2008.

38. Barbour M, Allan D, Xu H, Pei C, Chen M, Niedbala W, Fukada SY, Besnard AG, Alves-Filho JC, Tong X, et al: IL-33 attenuates the development of experimental autoimmune uveitis. Eur J Immunol 44: 3320-3329, 2014.

39. Besnard AG, Togbe D, Guillou N, Erard F, Quesniaux V and Ryffel B: IL-33-activated dendritic cells are critical for allergic airway inflammation. Eur J Immunol 41: 1675-1686, 2011.

40. Solinas G, Germano G, Mantovani A and Allavena P: Tumorassociated macrophages (TAM) as major players of the cancer-related inflammation. J Leukoc Biol 86: 1065-1073, 2009.

41. Dhillon AS, Hagan S, Rath O and Kolch W: MAP kinase signalling pathways in cancer. Oncogene 26: 3279-3290, 2007.
42. Schmieder A, Multhoff G and Radons J: Interleukin-33 acts as a pro-inflammatory cytokine and modulates its receptor gene expression in highly metastatic human pancreatic carcinoma cells. Cytokine 60: 514-521, 2012.

43. Markovic DS, Vinnakota K, Chirasani S, Synowitz M, Raguet H, Stock K, Sliwa M, Lehmann S, Kälin R, van Rooijen N, et al: Gliomas induce and exploit microglial MT1-MMP expression for tumor expansion. Proc Natl Acad Sci USA 106: 12530-12535, 2009.

44. Mentlein R, Hattermann $\mathrm{K}$ and Held-Feindt J: Lost in disruption: Role of proteases in glioma invasion and progression. Biochim Biophys Acta 1825: 178-185, 2012.

45. Zheng X, Chopp M, Lu Y, Buller B and Jiang F: MiR-15b and miR-152 reduce glioma cell invasion and angiogenesis via NRP-2 and MMP-3. Cancer Lett 329: 146-154, 2013. 